\title{
Características do ambiente físico e organizacional para a prática de atividade física nas escolas de Curitiba, Brasil
}

\author{
Characteristics of physical activity facilities in schools of Curitiba, Brazil
}

\section{AUTORES \\ Alexandre Augusto de Paula da Silva ${ }^{1}$ (D) Adalberto Aparecido dos Santos Lopes ${ }^{1,2}$ (IC Crisley Vanessa Prado ${ }^{1,3}$ (D) Adriano Akira Ferreira Hino ${ }^{1,4,5}$ (D) Rodrigo Siqueira Reis $^{1,6,7}$ (D) \\ 1 Pontifícia Universidade Católica do Paraná. Grupo de Pesquisa em Atividade Física e Qualidade de Vida, Curitiba, Paraná, Brasil. \\ 2 Universidade Federal do Paraná. Programa de Pós-Graduação em Educação Física. Curitiba, Paraná, Brasil. \\ 3 Universidade do Contestado. Curso de Graduação em Educação Física, Mafra, Santa Catarina, Brasil. \\ 4 Pontifícia Universidade Católica do Paraná. Programa de Pós-Graduação em Tecnologia em Saúde, Curitiba, Paraná, Brasil. \\ 5 Universidade Tecnológica Federal do Paraná, Programa de Pós-Graduação em Educação Física, Curitiba, Paraná, Brasil \\ 6 Pontifícia Universidade Católica do Paraná Programa de Pós-Graduação em Gestão Urbana, Curitiba, Paraná, Brasil. \\ 7 Washington University in St. Louis. Brown School, Prevention Research Center, Saint Louis, Missouri, Estados Unidos da América.}

\section{CONTATO}

Alexandre Augusto de Paula da Silva alexandre_03_19@hotmail.com

Pontifícia Universidade Católica do Paraná, Grupo de Pesquisa em Atividade Física e Qualidade de Vida.

Rua Imaculada Conceição, 1155, Prado

Velho, Curitiba, Paraná, Brasil.

CEP: 80215-901.

DOI

$10.12820 /$ rbafs.23e0027

\begin{abstract}
RESUMO
O objetivo deste estudo foi descrever as características das estruturas para a prática de atividade física (AF) entre escolas públicas e privadas de Curitiba, Brasil. Estudo transversal com 114 escolas (72,8\% públicas). Os respectivos professores de Educação Física $(E F)(n=114)$ das escolas informaram as características organizacionais. O ambiente escolar foi avaliado por meio de observação sistemática com uma ferramenta de auditoria para identificar o tipo, qualidade (não funcional, ruim, média e excelente) e quantidade de estruturas para AF. Os professores de EF reportaram a disponibilidade das estruturas por meio de um instrumento padronizado. Para análise, utilizou-se da distribuição de frequências, os testes de Qui-quadrado e U de Mann Whitney mantendo um nível de significância de 5\%. Nas escolas públicas, houve maior proporção das áreas para jogos com qualidade "média" (54,6\%), esportes "ruim" (29,1\%), quadras individuais "ruim" (38,7\%) e poliesportivas "não funcionais" (37,6\%), enquanto em escolas privadas eram de qualidade "excelente" as áreas para jogos $(68,9 \%)$, esportes $(57,3 \%)$, quadras individuais $(50,0 \%)$ e poliesportivas $(47,0 \%)$ com diferença significante em todas as áreas entre as escolas $(\mathrm{p}<0,001)$. Escolas públicas possuem aproximadamente um equipamento para AF a cada 100 alunos, enquanto a escolas privadas possuem o dobro $(p<0,001)$. A disponibilidade das estruturas foi, em geral, semelhante entre os tipos de escola, exceto no acesso a "playgrounds" e o espaço para prática de "tênis de mesa" no período do recreio foi maior em escolas privadas $(p<0,05)$. As escolas privadas apresentaram maior qualidade e quantidade de estruturas relacionadas à $\mathrm{AF}$ quando comparadas as escolas públicas.
\end{abstract}

Palavras-chave: Ambiente construído; Atividade motora; Escolas; Ambiente; Estudos transversais.

\section{ABSTRACT}

The aim of this study was to describe the characteristics of physical activity (PA) facilities and structure between public and private schools of Curitiba, Brazil. Cross-sectional study conducted in 114 schools (72.8\% public schools). A physical education (PE) instructor from each school $(n=114)$ reported about $P A$ and PE organizational characteristics. The school environment was assessed through systematic observation tool to identify type, quality (no functional, poor, medium and excellent) and number of PA facilities. All organizational characteristics were reported by PE instructors through a standardized questionnaire. Data was analyzed through frequencies, chi-square and Mann Whitney $U$ tests considering $5 \%$ of statistical significance. In public schools PA facilities for play were "medium" (54.6\%), sports (29.1\%), individual courts "poor" (38,7\%) and multi sports courts "no functional" (37.6\%), whereas private schools presented facilities with "excellent" quality for play areas (68.9\%), sports (57.3\%), individual courts (50.0\%) and multi sports courts (47.0\%) with significant difference in all areas between schools $(p<0.001)$. Public schools had one $P A$ equipment per 100 students, whereas private schools had twice as many equipments ( $p<0.001)$. The availability of $P A$ facilities was, in general, similar in both types of schools, except in access in playgrounds and table tennis areas during the recess, being higher in private schools $(p<0.05)$. Private schools showed greater quantity and higher quality of $P A$ facilities than public schools.

Keywords: Built environment; Motor activity; Schools; Environment; Cross-sectional studies. 


\section{Introdução}

Características físicas e sociais do ambiente podem contribuir na adoção de comportamentos ativos na população ${ }^{1}$. Em crianças e adolescentes, o ambiente escolar oferece importante oportunidade para a promoção $\mathrm{da}$ atividade física $(\mathrm{AF})^{2}$, uma vez que os jovens matriculados em instituições de ensino permanecem ao menos um período do dia (4,5 horas/dia) expostos a este ambiente, o qual em grande parte, é destinado para atividades com pouca ou nenhuma demanda energética ${ }^{3}$.

Programas efetivos para promoção de AF no contexto da escola devem ser compostos de professores bem preparados, currículos estruturados, materiais ${ }^{4}$, além da qualidade e disponibilidade de estruturas ${ }^{5}$. Apesar destas características, os estudos previamente realizados analisaram principalmente o engajamento dos alunos e o nível de AF nas aulas de Educação Física $(\mathrm{EF})^{6,7}$. Embora exista uma quantidade limitada de estudos sobre o ambiente escolar, a literatura tem apontado que, não somente a quantidade, mas também a qualidade das estruturas da escola têm se associado de forma positiva com maiores níveis de $\mathrm{AF}^{8}$. Em um estudo realizado nos Estados Unidos, estas características ambientais combinadas com a supervisão das atividades explicaram 42\% e 59\% da variância nos níveis de $\mathrm{AF}$ entre meninas e meninos, respectivamente ${ }^{9}$.

No Brasil, os resultados sobre este tema ainda são escassos e inconsistentes. Por exemplo, dados da Pesquisa Nacional de Saúde do Escolar (PeNSE), indicam uma associação positiva entre níveis de $\mathrm{AF}$ e disponibilidade de estruturas para $\mathrm{AF}^{10}$. No entanto, o estudo realizado no município de Passo Fundo, Rio Grande do Sul, por Dias et $\mathrm{al}^{11}$ encontrou associação inversa entre a quantidade de passos e a qualidade das estruturas da escola. Além da aparente inconsistência entre estes achados, as características das estruturas para AF foi descrita de forma limitada, sem detalhamento quanto ao perfil das escolas, como o tipo de administração escolar (pública ou privada). Apenas um estudo, realizado na cidade de Pelotas, Rio Grande do Sul, identificou tais características e demonstrou que escolas privadas tinham maior quantidade e qualidade de estruturas relacionadas à $\mathrm{AF}$ quando comparadas com as escolas públicas ${ }^{12}$.

Desta forma, descrever as condições do ambiente escolar para a AF pode auxiliar no planejamento e direcionamento de recursos para o aumento e melhoria das estruturas para a promoção da AF dentro do contexto escolar, bem como os programas de promoção de $\mathrm{AF}$ que empreguem espaços para a prática de $\mathrm{AF}$ entre seus elementos. Dessa forma, o presente estudo teve como objetivo descrever as características das estruturas para a prática de atividade física entre escolas públicas e privadas de Curitiba, Brasil.

\section{Métodos}

Este estudo de delineamento transversal e exploratório, é parte de um projeto denominado International Physical Activity and the Environment Network - IPEN ${ }^{13}$, o qual avaliou características relacionadas à prática de AF em adolescentes da cidade de Curitiba, Paraná entre agosto/2013 e maio/2014. Informações adicionais sobre o projeto podem ser encontradas na literatura ${ }^{3}$. O projeto foi aprovado pelo Comitê de Ética e Pesquisa da Pontifícia Universidade Católica do Paraná, parecer no $135-945 / 2012$.

As escolas que compuseram o estudo foram indicadas por 503 adolescentes participantes do IPEN. A seleção dos adolescentes participantes do estudo ocorreu em múltiplos estágios sendo no primeiro estágio selecionado setores censitários da cidade.

Em Curitiba, no período em que o estudo foi conduzido, existiam 2.395 setores censitários selecionados de acordo com as características de walkability e renda do bairro ${ }^{14}$. $\mathrm{O}$ escore de walkability foi criado a partir dos indicadores do uso do solo, densidade residencial e densidade de ruas ${ }^{3}$. Com o objetivo de maximizar a variabilidade de walkability e renda, os setores foram classificados em decis para ambas as variáveis e selecionados os setores pela combinação dos quatro extremos (baixo/alto) de walkability e renda. No total, foram selecionados intencionalmente 32 setores censitários, de maneira que os quatro grupos fossem representados (oito de cada grupo).

$\mathrm{O}$ arrolamento dos segmentos de rua foi realizado em todas as quadras dos setores censitários. Em caso de recusa da família ou de não residir algum adolescente, a próxima residência à esquerda foi abordada. Em cada domicílio foi selecionado um adolescente e o respectivo responsável. Os critérios para a seleção dos adolescentes foram: meninas mais jovens, meninos mais velhos, para permitir a seleção equitativa entre os sexos. Em caso de recusa do adolescente selecionado, outro adolescente da residência poderia ser escolhido. Foram incluídos adolescentes com idade entre 12 - 17 anos.

A partir dos adolescentes que completaram todas as etapas do inquérito domiciliar, foram identificadas 163 escolas, sendo $99(60,7 \%)$ da rede pública e $64(39,3 \%)$ 
da rede privada de ensino. Primeiramente, foi realizado o contato com a Secretaria Municipal de Educação de Curitiba e a Secretaria Estadual de Educação do Paraná, solicitando o acesso às escolas. Após a autorização, o diretor de cada instituição foi convidado a participar, mediante aos esclarecimentos sobre o projeto e suas etapas, com a anuência e assinatura do Termo de Consentimento Livre e Esclarecido (TCLE).

Foram incluídas no estudo apenas as escolas reportadas pelos adolescentes participantes do estudo e que no ano da coleta de dados apresentavam as seguintes caraterísticas: a) estivessem contidas no limite territorial da cidade de Curitiba, Paraná; b) ofertassem ensino fundamental e/ou médio e; c) que apresentassem a disciplina de EF como componente curricular.

A auditagem da escola foi realizada com a Ferramenta de Auditoria da Escola, instrumento adaptado do original, desenvolvido no Reino Unido ${ }^{15,16}$, com objetivo de avaliar as características do ambiente escolar relacionadas à prática de $\mathrm{AF}$ a partir da observação sistemática. A adaptação do instrumento original consistiu em tradução e adaptação dos itens, seguido de aplicação em seis escolas (três públicas e três particulares) para teste de "clareza". Após acrescentadas algumas estruturas que o instrumento não contemplava, foi realizada uma segunda aplicação em quatro escolas (duas públicas e duas privadas). A versão final do instrumento foi composta por 48 itens, divididos em cinco seções: 1) acesso; 2) área do entorno; 3) estética; 4) uso dos espaços e; 5) alimentação ${ }^{17}$. Para este estudo, foram utilizados somente os itens da seção quatro, caracterizados pelas áreas para jogos, áreas para esportes, quadras individuais e quadras poliesportivas.

A reprodutibilidade do instrumento foi testada de maneira independente por quatro avaliadores em duas escolas. As comparações entre avaliadores apresentaram fidedignidade elevada para as escalas "áreas para jogos" (CCI: 0,94; IC95\%: 0,80; 0,98) e "áreas para esportes" (CCI: 0,93; IC95\%: 0,76; 0,98), e moderada para as escalas "quadra individual" (CCI: - 0,56; IC95\%: - 4,40; - 0,55) e "quadra poliesportiva" (CCI: 0,23; IC95\%: - 1,66; 0,78) ${ }^{17}$. Os valores de IC negativos podem ser justificados pela dificuldade na avaliação das quadras sobrepostas (futsal, vôlei, handebol e basquetebol). Contudo, a média de estruturas entre a primeira e segunda avaliação apresentou pouca diferença nas duas medidas $(1,0$ e 1,16$)$.

Para descrição das características organizacionais da escola, foi utilizado o instrumento "Avaliação da
Promoção da AF na Escola”, adaptado do School Physical Activity Policy Assessment (S-PAPA) ${ }^{18}$. Foi realizada tradução e adaptação para o contexto brasileiro, seguidas de aplicação para teste de "clareza" dos itens em quatro professores (dois da rede pública e dois da rede particular de ensino).

O instrumento final foi composto por uma sessão de contextos gerais da escola, que contempla o número de alunos matriculados e níveis de ensino ofertados, assim como a presença e disponibilidade de estruturas relacionadas à prática de $\mathrm{AF}$ em diferentes momentos, divididos em: antes e depois da aula, durante o recreio e durante as aulas de educação física. Outros três módulos específicos compuseram o instrumento: módulo 1 (48 itens) - Características relacionadas à Educação Física; módulo 2 (33 itens) - Características relacionadas ao recreio; módulo 3 (25 itens) - Características relacionadas às atividades extracurriculares. Para este estudo, foi utilizado apenas a sessão de contextos gerais da escola.

A versão brasileira do instrumento foi denominada "Avaliação da Promoção da Atividade Física na Escola” - (APAFE), e foi aplicado por meio de uma entrevista realizada com um professor de EF da escola, selecionado por conveniência. A reprodutibilidade do APAFE apresentou valores semelhantes ao instrumento original, sendo considerados "moderados" a "elevados” (Kappa 0,47-0,81), e percentual de concordância acima de $80 \%{ }^{17}$.

As estruturas para a prática de AF disponíveis dentro do perímetro da escola foram analisadas segundo seu tipo, qualidade e quantidade. Inicialmente, as estruturas foram classificadas segundo a seguinte tipologia: a) área para jogos — voltadas para brincadeiras e atividades lúdicas (área pavimentada ao ar livre; estação de exercício; gramado ou superfície macia; marca para jogos; playgrounds e; outros, que inclui sala de motricidade ou salas de xadrez); b) áreas para esportes - espaços que possuem regras e objetivos mais específicos da prática de esportes, individual ou coletivo (campo de futebol; cancha de futebol; cancha de voleibol; piscina; pista de corrida; sala de dança; sala de ginástica; sala de lutas; tênis de mesa e; outros, como sala de musculação e sala de spinning); c) quadras individuais - espaços para a prática de apenas um tipo de esporte, consideradas aquelas contidas em locais sem cobertura, com cobertura e dentro de um ginásio (basquetebol, futsal, handebol, tênis e voleibol) e; d) quadras poliesportivas - espaços para a prática de mais de um tipo de espor- 
te, consideradas aquelas contidas em local sem cobertura, com cobertura e dentro de um ginásio (basquetebol, futsal, handebol, tênis e voleibol). Em seguida, a qualidade das estruturas presentes foi classificada em quatro níveis: a) não funcional (estruturas existentes, mas não utilizáveis); b) ruim (estrutura não apresenta algum recurso ou está danificada); c) média (estrutura com quase todos os recursos para ser utilizada, em boas condições); d) excelente (estrutura com condição perfeita de uso). Para permitir a comparação entre a quantidade de estruturas utilizáveis, a variável também foi classificada em "não funcional" e "funcional" (qualidade ruim, média e excelente). A quantidade das estruturas foi contabilizada de acordo com a frequência observada das estruturas dentro do terreno da escola, exceto as quadras poliesportivas, onde cada demarcação para um esporte específico foi contada como uma quadra.

$\mathrm{O}$ acesso aos locais para a AF na escola foi determinado a partir do relato dos professores de EF. Para tanto, os professores foram entrevistados sobre a agenda de utilização das estruturas (ginásio, quadra pavimentada sem cobertura, quadra pavimentada coberta, campo de futebol, cancha de futebol, cancha de voleibol, pista de corrida, área pavimentada, playgrounds, tênis de mesa, piscina, sala de ginástica artística e sala de lutas) durante as aulas de EF, recreio e períodos antes e depois da aula. As respostas foram classificadas em "não" para uso não permitido e "sim" para uso permitido em cada um dos períodos.

O tipo de escola foi categorizado pela forma de administração da instituição, em "pública", gerida por um órgão governamental e "privada", gerida por uma empresa privada ou grupo educacional.

A entrada de dados foi realizada no software Epidata com dupla digitação, permitindo o controle e correção dos erros. A descrição das estruturas para prática de AF foi realizada de acordo com o tipo de local, qualidade (não funcional, ruim, média, excelente) e disponibilidade, utilizando a distribuição de frequências absoluta e relativa e os testes de qui-quadrado (heterogeneidade e tendência linear) e ainda o teste exato de Fisher para comparação das variáveis categóricas entre as escolas públicas e privadas. A quantidade de estruturas foi avaliada segundo a proporção das mesmas em relação ao número de alunos na escola e a funcionalidade para o uso (unidade a cada 100 alunos). Este indicador permitiu a comparação entre escolas de diferentes portes. A não-normalidade de distribuição das variáveis dependentes foi verificada com teste de Kolmogorov Smirnov, sendo, portanto, adotados procedimentos não paramétricos, tanto para descrição quanto para a comparação entre escolas públicas e privadas (teste U-Mann Whit$n e y)$. As análises foram realizadas no software SPSS 20.0 e o nível de significância mantido em $5 \%$.

\section{Resultados}

Entre as 163 escolas identificadas, sete (4,3\%) eram inelegíveis por não apresentaram EF na grade curricular, e três $(1,8 \%)$ por não estarem localizadas na cidade de Curitiba, Paraná. Além disso, 29 (17,8\%) recusaram ou não responderam ao contato inicial para participar do estudo (5,5\% públicas e $12,9 \%$ privadas). Foram ainda excluídas 10 escolas com dados incompletos de auditagem do ambiente escolar ou de entrevistas. A amostra analítica foi composta de 114 escolas (72,8\% públicas).

As escolas privadas ofertavam em maior proporção a educação infantil $(76,0 \%$; $<<0,001)$ e ensino fundamental I $(66,7 \% ; \mathrm{p}<0,001)$. As escolas públicas possuíam em maior proporção a oferta de ensino fundamental II $(88,6 \% ; p=0,041)$. Ao menos um recreio era ofertado por período de aula (92,0\%), sendo que, as escolas privadas apresentaram em maior proporção dois ou mais recreios por turno $(22,6 \% ; \mathrm{p}=0,002)$ com maior duração $(74,2 \% ; p=0,003)$. A maior parte das escolas estava localizada em setores censitários de renda elevada $(69,3 \%)$, com mais de 1.000 alunos matriculados (43,0\%). O número de professores de $\mathrm{EF}$ presentes em cada escola variou entre 1 - $3(35,4 \%)$ e 4 - $5(35,4 \%)$ em sua maioria, com tempo de trabalho na escola menor ou igual a três anos (39,3\%). Oito a cada dez escolas ofereciam atividades esportivas de contraturno $(81,2 \%)$ - Tabela 1.

Em geral, as escolas públicas, mostraram maiores proporções de áreas para jogos de qualidade "média" (54,6\%), áreas para esportes de qualidade "ruim" $(29,1 \%)$, quadras individuais "ruim" $(38,7 \%)$ e de quadras poliesportivas classificadas com "não funcionais" $(37,6 \%)$. Já as escolas privadas, apresentaram maior proporção de estruturas com qualidade "excelente" nas áreas para jogos $(68,9 \%)$, áreas para esportes $(53,7 \%)$, quadras individuais $(50,0 \%)$ e quadras poliesportivas (47,0\%). Quando comparadas as proporções de qualidade das áreas entre escolas públicas e privadas, houve diferença estatística significante nas áreas para jogos, esportes, quadras individuais e poliesportivas ( $\mathrm{p}$ $<0,001)$ - Figura 1.

A mediana de áreas para jogos (para cada 100 alu- 
Tabela 1 - Características descritivas das escolas segundo o tipo de administração. Curitiba, Paraná, Brasil, 2013-2014 (n = 114).

\begin{tabular}{|c|c|c|c|c|c|c|c|}
\hline \multirow[t]{2}{*}{ Variável } & \multicolumn{2}{|c|}{$\begin{array}{c}\text { Total } \\
(\mathrm{n}=114)\end{array}$} & \multicolumn{2}{|c|}{$\begin{array}{c}\text { Pública } \\
(\mathrm{n}=83 ; 72,8 \%)\end{array}$} & \multicolumn{2}{|c|}{$\begin{array}{c}\text { Privada } \\
(\mathrm{n}=31 ; 27,2 \%)\end{array}$} & \multirow[b]{2}{*}{$\mathrm{p}$} \\
\hline & $\mathrm{n}$ & $\%$ & $\mathrm{n}$ & $\%$ & $\mathrm{n}$ & $\%$ & \\
\hline \multicolumn{8}{|c|}{ Renda do setor censitário (decis) } \\
\hline Baixo (1 - 5) & 35 & 30,7 & 28 & 33,7 & 7 & 22,6 & \multirow[t]{2}{*}{$0,251^{\mathrm{h}}$} \\
\hline Elevado $(6-10)$ & 79 & 69,3 & 55 & 66,3 & 24 & 77,4 & \\
\hline \multicolumn{8}{|l|}{ Número de alunos } \\
\hline Até 500 & 24 & 21,0 & 16 & 19,3 & 8 & 25,8 & \multirow[t]{3}{*}{$0,191^{\mathrm{t}}$} \\
\hline Entre 501 e 1.000 & 41 & 36,0 & 28 & 33,7 & 13 & 41,9 & \\
\hline Acima de 1.000 & 49 & 43,0 & 39 & 47,0 & 10 & 32,3 & \\
\hline \multicolumn{8}{|c|}{ Níveis de ensino ofertado } \\
\hline \multicolumn{8}{|l|}{ Educação infantil } \\
\hline Não & 75 & 77,3 & 69 & 95,8 & 6 & 24,0 & \multirow[t]{2}{*}{$<0,001^{\mathrm{f}}$} \\
\hline Sim & 22 & 22,7 & 3 & 4,2 & 19 & 76,0 & \\
\hline \multicolumn{8}{|l|}{ Ensino fundamental I } \\
\hline Não & 64 & 68,1 & 56 & 80,0 & 8 & 33,3 & \multirow[t]{2}{*}{$<0,001^{\mathrm{h}}$} \\
\hline $\operatorname{Sim}$ & 30 & 31,9 & 14 & 20,0 & 16 & 66,7 & \\
\hline \multicolumn{8}{|l|}{ Ensino fundamental II } \\
\hline Não & 15 & 16,0 & 8 & 11,4 & 7 & 29,2 & \multirow[t]{2}{*}{$0,041^{\mathrm{h}}$} \\
\hline $\operatorname{Sim}$ & 79 & 84,0 & 62 & 88,6 & 17 & 70,8 & \\
\hline \multicolumn{8}{|l|}{ Ensino médio } \\
\hline Não & 22 & 23,4 & 19 & 27,1 & 3 & 12,5 & \multirow[t]{2}{*}{$0,173^{\mathrm{f}}$} \\
\hline $\operatorname{Sim}$ & 72 & 76,6 & 51 & 72,9 & 21 & 87,5 & \\
\hline \multicolumn{8}{|c|}{ Professores de EF na escola } \\
\hline $1-3$ professores & 40 & 35,4 & 24 & 29,3 & 16 & 51,6 & \multirow[t]{3}{*}{$0,183^{t}$} \\
\hline $4-5$ professores & 40 & 35,4 & 34 & 41,5 & 6 & 19,4 & \\
\hline$\geq 6$ professores & 33 & 29,2 & 24 & 29,3 & 9 & 29,0 & \\
\hline \multicolumn{8}{|c|}{ Tempo de trabalho dos professores na escola } \\
\hline$\leq 3$ anos & 37 & 39,3 & 30 & 42,9 & 7 & 29,2 & \multirow[t]{3}{*}{$0,415^{\mathrm{t}}$} \\
\hline Entre 3 a 9 anos & 31 & 33,0 & 21 & 30,0 & 10 & 41,7 & \\
\hline$>9$ anos & 26 & 27,7 & 19 & 27,1 & 7 & 29,2 & \\
\hline \multicolumn{8}{|c|}{ Oferta de atividades esportivas no contraturno escolar } \\
\hline Não & 18 & 18,8 & 15 & 22,4 & 3 & 10,3 & \multirow[t]{2}{*}{$0,255^{\mathrm{f}}$} \\
\hline $\operatorname{Sim}$ & 78 & 81,2 & 52 & 77,6 & 26 & 89,7 & \\
\hline \multicolumn{8}{|l|}{ Recreio } \\
\hline Quantidade de recrei & & & & & & & \\
\hline Um & 104 & 92,0 & 80 & 97,6 & 24 & 77,4 & $0,002^{f}$ \\
\hline Dois ou mais & 9 & 8,0 & 2 & 2,4 & 7 & 22,6 & \\
\hline Duração dos recreios & & & & & & & \\
\hline$\leq 15$ minutos & 55 & 48,7 & 47 & 57,3 & 8 & 25,8 & $0 \cap 03^{\mathrm{h}}$ \\
\hline$>15$ minutos & 58 & 51,3 & 35 & 42,7 & 23 & 74,2 & 0,003 \\
\hline
\end{tabular}

$\mathrm{h}=$ Qui-quadrado para heterogeneidade; $\mathrm{t}=$ Qui-quadrado para tendência linear; $\mathrm{f}=$ Teste exato de Fisher; $\mathrm{p}<0,05$.

nos), classificadas como "funcionais" foi maior entre as escolas privadas $(0,96)$ quando comparado as escolas públicas $(0,42 ; \mathrm{p}<0,001)$. Da mesma forma, foi observada diferença estatisticamente significante no número de áreas funcionais para esportes $(\mathrm{p}<0,001)$, quadras individuais $(\mathrm{p}<0,001)$ e poliesportivas $(\mathrm{p}<$ $0,001)$ Apenas nas quadras individuais "não funcionais" as escolas privadas apresentaram maior número $(0,32)$ quando comparadas com as escolas públicas $(0,24 ; \mathrm{p}=$ 0,030). Ao considerar o número total de estruturas, foi observada diferença entre o número de equipamentos "funcionais" para cada 100 alunos entre escolas públicas $(0,95)$ e privadas $(2,18 ; \mathrm{p}<0,001)$ - Figura 2 . 


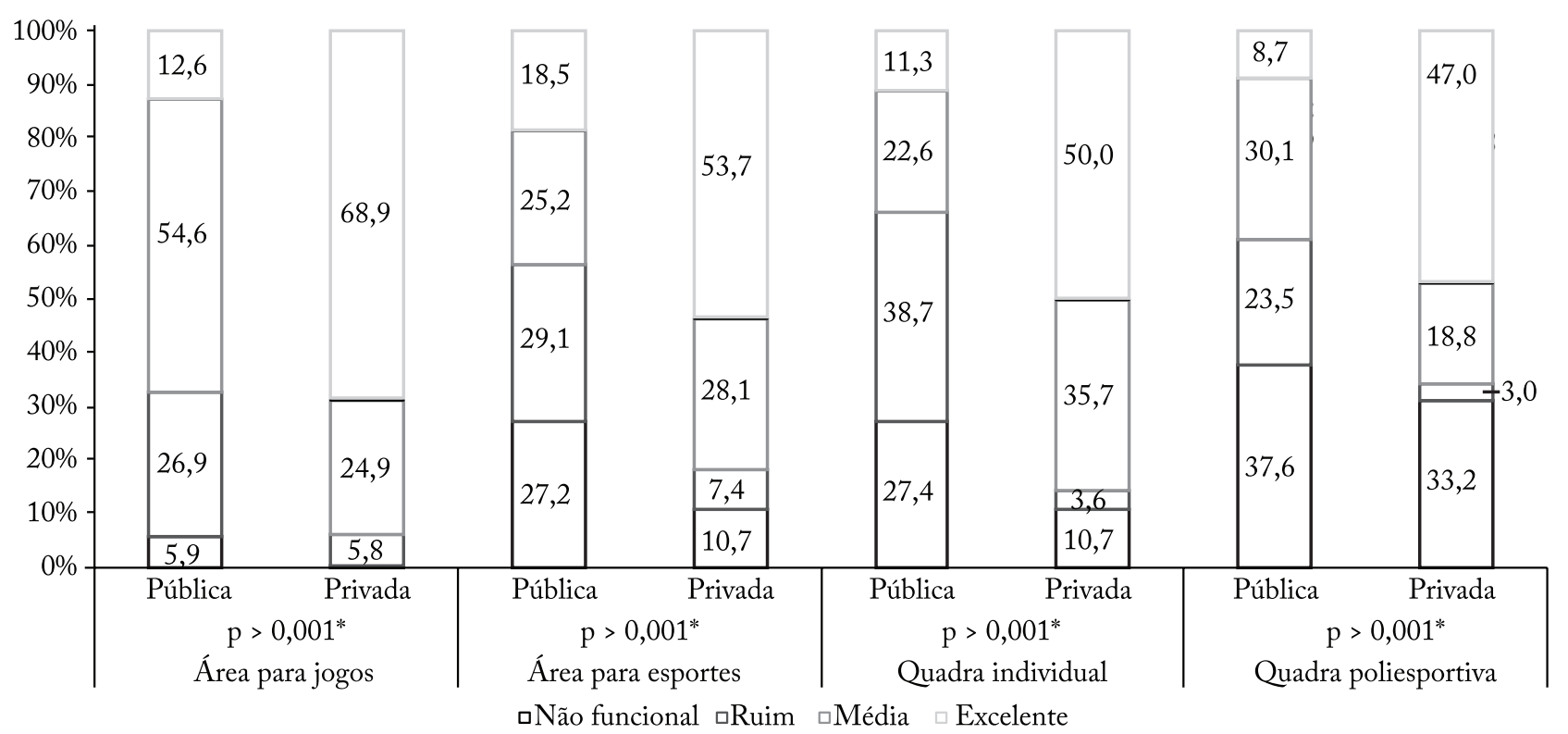

Figura 1 - Proporção das estruturas para prática de AF da escola, segundo sua funcionalidade e o tipo de administração. Curitiba, Brasil, 2013-2014 ( $=114)$.

Teste de Qui-quadrado para tendência; * $\mathrm{p}<0,001$.

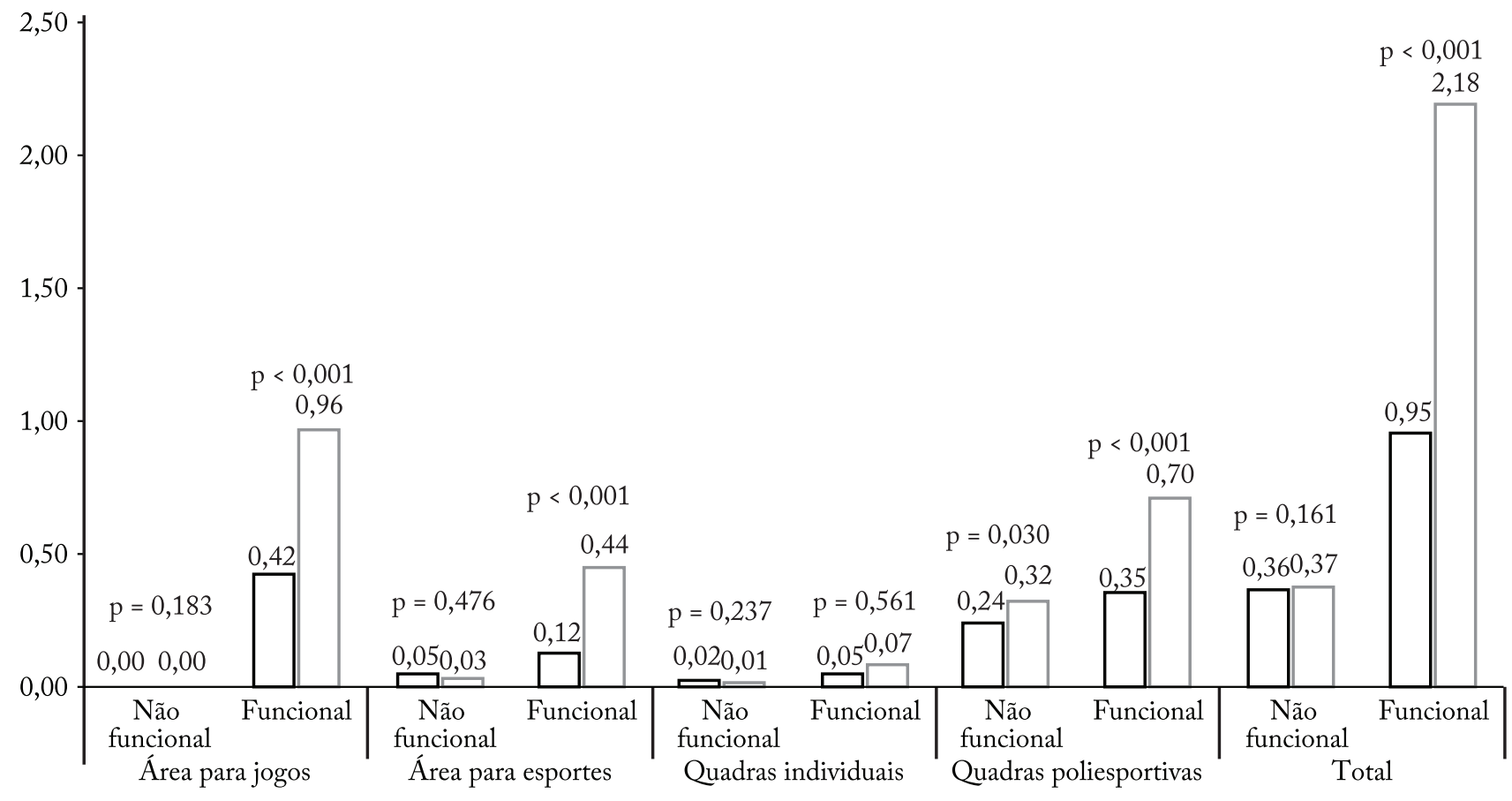

$\square$ Pública $\square$ Privada

Figura 2 - Comparação da mediana da quantidade das estruturas não funcionais e funcionais, para cada 100 alunos nas escolas, entre o tipo de administração. Curitiba, Brasil $(\mathrm{n}=114)$.

Teste U de Mann Whitney; *p < 0,05.

A disponibilidade das estruturas para a prática de AF durante a EF, recreio e antes/depois da aula foi semelhante nas escolas públicas e privadas, onde grande parte permitiu a utilização principalmente nas aulas de EF (> 55,6\% - 100\%). Apenas a utilização das es- truturas "playgrounds" $(\mathrm{p}=0,027)$ e "tênis de mesa" ( $\mathrm{p}$ $=0,007)$ durante o recreio apresentou uma diferença significativa entre as escolas, com maiores proporções entre as privadas - Tabela 2 . 
Tabela 2 - Disponibilidade dos locais para a prática de AF em períodos específicos, segundo o tipo de administração. Curitiba, Paraná, Brasil, 2013-2014 ( $\mathrm{n}=114)$

\begin{tabular}{|c|c|c|c|c|c|c|c|}
\hline \multirow{2}{*}{ Locais } & \multicolumn{2}{|c|}{ Total } & \multicolumn{2}{|c|}{ Pública } & \multicolumn{2}{|c|}{ Privada } & \multirow[b]{2}{*}{$\mathrm{p}$} \\
\hline & $\mathrm{n}$ & $\%$ & $\mathrm{n}$ & $\%$ & $\mathrm{n}$ & $\%$ & \\
\hline \multicolumn{8}{|l|}{ Ginásio } \\
\hline $\mathrm{EF}$ & 51 & 96,2 & 27 & 93,1 & 24 & 100,0 & $0,495^{\mathrm{f}}$ \\
\hline Recreio & 40 & 75,5 & 20 & 69,0 & 20 & 83,3 & $0,338^{f}$ \\
\hline Antes/depois da aula & 40 & 75,5 & 20 & 69,0 & 20 & 83,3 & $0,338^{f}$ \\
\hline \multicolumn{8}{|c|}{ Quadra pavimentada sem cobertura } \\
\hline $\mathrm{EF}$ & 80 & 100,0 & 64 & 100,0 & 16 & 100,0 & \\
\hline Recreio & 70 & 87,5 & 56 & 87,5 & 14 & 87,5 & $1,000^{f}$ \\
\hline Antes/depois da aula & 53 & 66,2 & 42 & 65,6 & 11 & 68,8 & $0,813^{\mathrm{h}}$ \\
\hline \multicolumn{8}{|c|}{ Quadra pavimentada com coberta } \\
\hline $\mathrm{EF}$ & 67 & 98,5 & 51 & 98,1 & 16 & 100,0 & $1,000^{f}$ \\
\hline Recreio & 58 & 85,3 & 43 & 82,7 & 15 & 93,8 & $0,432^{\mathrm{f}}$ \\
\hline Antes/depois da aula & 42 & 61,8 & 33 & 63,5 & 9 & 56,2 & $0,604^{\mathrm{h}}$ \\
\hline \multicolumn{8}{|l|}{ Campo de futebol } \\
\hline $\mathrm{EF}$ & 16 & 94,1 & 7 & 87,5 & 9 & 100,0 & $0,471^{\mathrm{f}}$ \\
\hline Recreio & 8 & 47,1 & 2 & 25,0 & 6 & 66,7 & $0,153^{\mathrm{f}}$ \\
\hline Antes/depois da aula & 5 & 29,4 & 1 & 12,5 & 4 & 44,4 & $0,149^{f}$ \\
\hline \multicolumn{8}{|l|}{ Cancha de futebol } \\
\hline $\mathrm{EF}$ & 11 & 100,0 & 8 & 100,0 & 3 & 100,0 & \\
\hline Recreio & 10 & 90,9 & 7 & 87,5 & 3 & 100,0 & $1,000^{f}$ \\
\hline Antes/depois da aula & 8 & 72,7 & 6 & 75,0 & 2 & 66,7 & $1,000^{f}$ \\
\hline \multicolumn{8}{|l|}{ Cancha de voleibol } \\
\hline $\mathrm{EF}$ & 14 & 100,0 & 12 & 100,0 & 2 & 100,0 & \\
\hline Recreio & 12 & 85,7 & 10 & 83,3 & 2 & 100,0 & $1,000^{\mathrm{f}}$ \\
\hline Antes/depois da aula & 8 & 72,7 & 6 & 75,0 & 2 & 66,7 & $1,000^{f}$ \\
\hline \multicolumn{8}{|l|}{ Pista de corrida } \\
\hline $\mathrm{EF}$ & 13 & 100,0 & 6 & 100,0 & 7 & 100,0 & \\
\hline Recreio & 9 & 69,2 & 3 & 50,0 & 6 & 85,7 & $0,545^{f}$ \\
\hline Antes/depois da aula & 5 & 41,7 & 1 & 16,7 & 4 & 66,7 & $0,242^{\mathrm{f}}$ \\
\hline \multicolumn{8}{|l|}{ Área pavimentada } \\
\hline $\mathrm{EF}$ & 86 & 81,9 & 61 & 79,2 & 25 & 89,3 & $0,385^{\mathrm{f}}$ \\
\hline Recreio & 100 & 95,2 & 75 & 97,4 & 25 & 89,3 & $0,117^{\mathrm{f}}$ \\
\hline Antes/depois da aula & 85 & 84,2 & 63 & 85,1 & 22 & 81,5 & $0,656^{\mathrm{f}}$ \\
\hline \multicolumn{8}{|l|}{ Playgrounds } \\
\hline $\mathrm{EF}$ & 25 & 75,8 & 6 & 54,5 & 19 & 86,4 & $0,088^{f}$ \\
\hline Recreio & 19 & 57,6 & 3 & 27,3 & 16 & 72,7 & $0,027^{\mathrm{f}}$ \\
\hline Antes/depois da aula & 14 & 43,8 & 2 & 18,2 & 12 & 57,1 & $0,061^{\mathrm{f}}$ \\
\hline \multicolumn{8}{|l|}{ Tênis de mesa } \\
\hline $\mathrm{EF}$ & 96 & 96,0 & 73 & 94,8 & 23 & 100,0 & $0,572^{\mathrm{f}}$ \\
\hline Recreio & 56 & 56,0 & 37 & 48,1 & 19 & 82,6 & $0,007^{\mathrm{f}}$ \\
\hline Antes/depois da aula & 34 & 34,7 & 25 & 32,9 & 9 & 40,9 & $0,487^{\mathrm{h}}$ \\
\hline \multicolumn{8}{|l|}{ Piscina } \\
\hline $\mathrm{EF}$ & 5 & 55,6 & 2 & 66,7 & 3 & 50,0 & $1,000^{f}$ \\
\hline Recreio & 1 & 11,1 & 1 & 33,3 & 0 & 0,0 & $0,375^{f}$ \\
\hline Antes/depois da aula & 1 & 12,5 & 1 & 33,3 & 0 & 0,0 & $0,375^{\mathrm{f}}$ \\
\hline \multicolumn{8}{|l|}{ Sala de ginástica artística } \\
\hline $\mathrm{EF}$ & 17 & 94,4 & 6 & 100,0 & 11 & 91,7 & $1,000^{f}$ \\
\hline Recreio & 3 & 17,6 & 0 & 0,0 & 3 & 25,0 & $0,515^{f}$ \\
\hline Antes/depois da aula & 3 & 17,6 & 0 & 0,0 & 3 & 25,0 & $0,515^{f}$ \\
\hline \multicolumn{8}{|l|}{ Sala de lutas } \\
\hline $\mathrm{EF}$ & 30 & 96,8 & 8 & 88,9 & 22 & 100,0 & $0,300^{f}$ \\
\hline Recreio & 3 & 9,7 & 0 & 0,0 & 3 & 13,6 & $0,534^{f}$ \\
\hline Antes/depois da aula & 4 & 13,3 & 0 & 0,0 & 4 & 19,0 & $0,287^{\mathrm{f}}$ \\
\hline
\end{tabular}

$\mathrm{F}=$ Teste exato de Fisher; $\mathrm{h}=$ Qui-quadrado para heterogeneidade ${ }^{*} \mathrm{p}<0,05$. 


\section{Discussão}

O presente estudo reúne informações sobre as estruturas para a prática de AF nas escolas. A avaliação qualitativa e quantitativa das instalações, assim como a disponibilidade de uso, permite identificar características do ambiente escolar, em relação a estruturas e espaços adequados, que podem auxiliar na promoção da AF em escolares. Ainda, considerar a quantidade de alunos na escola de acordo com o número de estruturas disponíveis permite compreender a distribuição e consequentemente oportunidade de acesso a equipamentos e espaços para a prática de AF.

Os principais achados apontam principalmente as disparidades em relação à qualidade e quantidade das estruturas para a prática de AF de acordo com o tipo de administração escolar. Enquanto que mais da metade das estruturas das escolas privadas eram de excelente qualidade, as escolas públicas apresentam uma a cada três com qualidade "ruim", considerando que os componentes ambientais da escola para a prática de AF têm sido associados a maiores níveis de $\mathrm{AF}$ dos escolares $^{5,8,19}$. A cobertura do solo com a presença de playgrounds, equipamentos para jogos e equipamentos de sala de EF também tem apresentado efeitos positivos na $\mathrm{AF}$ realizada no período de recreio e também nas aulas de $\mathrm{EF}^{20}$.

A maior proporção das áreas presentes em escolas públicas apresentou qualidade "ruim" (esportes e quadras individuais) "média" (jogos) e "não funcional" (quadras poliesportivas), enquanto a maior parte das áreas em escolas privadas foi classificada como de qualidade "excelente". Evidências mostram que qualidade das estruturas físicas das escolas pode influenciar na organização e oferta de $\mathrm{AF}$ no âmbito curricular ou extracurricular ${ }^{4,5}$. Por exemplo, a falta de estruturas ou equipamentos não adequados podem se tornar uma barreira para o bom andamento das aulas de $\mathrm{EF}^{21}$, principalmente em escolas públicas ${ }^{22,23}$. Esta informação reflete na EF escolar com soluções provisórias e instalações improvisadas ${ }^{22}$, que acabam por não suprir as necessidades formais e essenciais para a realização das aulas de qualidade, além da limitação para implementação de programas para promoção de $\mathrm{AF}^{23}$. Estas limitações estruturais requerem uma análise aprofundada, como a disponibilidade de verbas e de espaços para uso, principalmente em escolas públicas. É importante ressaltar que, apesar da menor proporção, as escolas privadas também apresentaram estruturas não funcionais, o que indica não ser uma característica ex- clusiva de escolas públicas. Aspectos da cultura esportiva do Brasil podem explicar estes resultados, uma vez que atividades estruturadas, supervisionadas e jogos em equipes são tradicionalmente realizados nas escolas, assim, os espaços para estes tipos de atividades são priorizados ${ }^{11}$.

Em relação à quantidade, escolas privadas apresentaram maior quantidade de estruturas "funcionais" para cada 100 alunos (jogos, esportes, individuais e poliesportivas). Também foi observado que nas quadras individuais, escolas privadas possuem mais estruturas "não funcionais". Quando considerado o total, haviam 0,95 estruturas para escolas públicas e 2,18 para privadas. Isso demonstra uma diferença importante entre os dois tipos de administração escolar. Considerando que, neste estudo, o número de alunos da maioria das escolas varia de 500 a mais de 1.000 , a baixa presença das estruturas para AF em escolas públicas parece não atender a demanda. É comum em algumas escolas que duas ou mais turmas dividam o mesmo espaço para as aulas de $\mathrm{EF}^{22}$. Neste sentido, as escolas deveriam dispor de mais espaços para que todas as turmas envolvidas das aulas de EF possam participar efetivamente das aulas práticas e atender a demanda de forma efetiva ${ }^{24}$. Um estudo realizado em Pelotas, Rio Grande do Sul também identificou que instituições privadas apresentaram maior quantidade de estruturas para $\mathrm{AF}^{12}$, de forma que alunos da rede privada de ensino estão expostos a um ambiente mais favorável para a prática de $\mathrm{AF}^{25,26}$. A UNESCO reporta a necessidade de recursos para espaços, instalações e equipamentos adequados para o desenvolvimento de aulas de EF de qualidade ${ }^{27}$. Ainda, alguns estudos sugerem que quando isoladas, as instalações não apresentaram associação significativa com a AF, porém quando somadas, resultaram em maiores níveis de $\mathrm{AF}$ entre os adolescentes ${ }^{8,28}$.

A disponibilidade das estruturas para as aulas de EF foi observada na maioria das escolas. Entretanto, alguns locais e instalações não poderiam ser utilizados no momento do recreio e antes/depois das aulas. Nas escolas públicas, existe uma precaução das equipes de coordenação da escola com a conservação das estruturas e equipamentos, principalmente pela dificuldade e demora na aquisição. De fato, apenas a presença da estrutura não reflete que os escolares se apropriem delas. Pesquisas apontam que o incentivo ao uso desses locais é de extrema importância para a promoção da $\mathrm{AF}^{20}$. Neste sentido, a avaliação da Efetividade dos Programas de AF no Brasil apresenta como uma das 
estratégias de promoção da AF nas escolas, o acesso a equipamentos e materiais ${ }^{29}$. Em contraponto, apenas o "playground" e "tênis de mesa" poderiam ser utilizados durante o recreio em maior proporção nas escolas privadas, em relação às públicas. Este resultado é importante, pois as intervenções que adicionaram materiais e equipamentos de recreio apresentaram um aumento significativo na $\mathrm{AF}$ dos escolares ${ }^{30}$.

Algumas limitações devem ser consideradas para a interpretação adequada dos resultados. A avaliação de qualidade e quantidade das estruturas foi feita por observação sistemática, o que poderia ser influenciada de acordo com as características do clima, tornando-a não funcional, ou a não visibilidade de materiais que poderiam ser desmontados e reposicionados no momento da avaliação. Em relação ao acesso as estruturas, as informações foram reportadas pelo professor de $\mathrm{EF}$, portanto viés de resposta não pode ser desconsiderado, uma vez que o tempo como servidor na escola poder afetar o conhecimento sobre as características existentes no estabelecimento. Por fim, o estudo não possui uma amostra representativa das escolas, o que não permite extrapolar os dados apresentados, já que as mesmas foram selecionadas em função de informações anteriormente obtidas.

No presente estudo as escolas privadas apresentaram melhor qualidade e maior quantidade de estruturas relacionadas à prática de $\mathrm{AF}$. Os resultados indicam que escolas públicas devem identificar a aprimorar as estruturas não funcionais e de baixa qualidade, considerando que a quantidade de estruturas disponíveis é menor. Tanto escolas públicas quanto privadas devem ampliar a disponibilidade de períodos de uso e a organização de programas estruturados de $\mathrm{AF}$ por gestores, diretores das escolas e professores de educação física, como atividades extracurriculares, de contraturno e recreios, de maneira a oportunizar maior participação de crianças e adolescentes e atividades físicas ${ }^{4}$. Futuros estudos devem identificar a quantidade necessária de estruturas destinadas à prática de $\mathrm{AF}$ nas escolas, bem como analisar as relações dos níveis de $\mathrm{AF}$ com a quantidade e qualidade dos espaços e equipamentos.

\section{Conflito de interesses}

Os autores declaram não haver conflito de interesses.

\section{Financiamento}

Apoio financeiro do National Institutes of Health (NIH).

\section{Contribuição dos autores}

Silva AAP, participou da coleta de dados concepção inicial do estudo, análise de dados, revisão da literatura e redação do manuscrito em todas as fases. Prado CV, participou da concepção inicial do estudo, revisão da literatura, coleta de dados e redação do manuscrito. Lopes AAS, participou da concepção inicial do estudo e redação do manuscrito. AAF Hino participou da concepção inicial do estudo, análise de dados e redação do manuscrito. RS Reis, foi responsável pela concepção e coordenação do projeto e participou da revisão crítica do manuscrito. Todos os autores aprovaram a versão final do artigo.

\section{Agradecimentos}

Os autores agradecem aos membros do Grupo de Pesquisa em Atividade Física e Qualidade de Vida (GPAQ/PUCPR) pela colaboração na coleta de dados. A Secretaria Municipal de Educação (SME) e Secretaria de Estado da Educação (SEED) e as respectivas escolas participantes do estudo.

\section{Referências}

1. Bauman AE, Reis RS, Sallis JF, Wells JC, Loos RJF, Martin BW. Correlates of physical activity: why are some people physically active and others not? The Lancet. 2012;380(9838):258-71.

2. Hoehner CM, Ribeiro IC, Parra DC, Reis RS, Azevedo MR, Hino AA, et al. Physical activity interventions in Latin America: expanding and classifying the evidence. Am J Prev Med. 2013;44(3):e31-40.

3. Alberico CO, Schipperijn J, Reis RS. Use of global positioning system for physical activity research in youth: ESPACOS Adolescentes, Brazil. Prev Med. 2016;103(S):59-65.

4. Ribeiro IC, Parra DC, Hoehner CM, Soares J, Torres A, Pratt M, et al. School-based physical education programs: evidence-based physical activity interventions for youth in Latin America. Glob Health Promot. 2010;17(2):5-15.

5. Fein AJ, Plotnikoff RC, Wild TC, Spence JC. Perceived environment and physical activity in youth. Int J Behav Med. 2004;11(3):135-42.

6. Hino AAF, Reis RS, Rodriguez-Añez CRR. Observação dos níveis de atividade física, contexto das aulas e comportamento do professor em aulas de educação física do ensino médio da rede pública. Rev Bras Ativ Fís Saúde. 2007;12(3):21-30.

7. Hallal PC, Knuth AG, Cruz DKA, Mendes MI, Malta DC. Prática de atividade física em adolescentes brasileiros. Cien Saude Colet. 2010;15(2):3035-42.

8. Knuth AG, Hallal PC. School environment and physical activity in children and adolescents: systematic review. Rev Bras Ativ Fís Saúde. 2012;17(1):463-73.

9. Sallis JF, Conway TL, Prochaska JJ, McKenzie TL, Marshall SJ, Brown $\mathrm{M}$. The association of school environments with youth physical activity. Am J Public Health. 2001;91(4):618-20.

10. Rezende LFM, Azeredo CM, Silva KS, Claro RM, FrançaJunior I, Peres MFT, et al. The role of school environment in physical activity among brazilian adolescents. PLoS One. 2015;10(6): 0131342.

11. Dias AF, Lemes VB, Brand C, Mello JB, Gaya AR, Gaya ACA. Association between school structure and physical activity in physical education class and school recess. Rev Bras Cineantropom Desempenho Hum. 2017;19(2):164-73. 
12. Knuth AG, Silva ICM, Hallal PC. Description of the school environment related to physical education classes, recess, extracurricular activities and physical spaces in the city of Pelotas, RS, Brazil. Rev Bras Ativ Fís Saúde. 2015;20(5):52433.

13. Sallis JF, Cerin E, Conway TL, Adams MA, Frank LD, Pratt $\mathrm{M}$, et al. Physical activity in relation to urban environments in 14 cities worldwide: a cross-sectional study. Lancet. 2016;387(10034):2207-17.

14. Reis RS, Hino AAF, Rech CR, Kerr J, Hallal PC. Walkability and Physical Activity: Findings from Curitiba, Brazil. Am J Prev Med. 2013;45(3):269-75.

15. Jones NR, Jones A, van Sluijs EM, Panter J, Harrison F, Griffin SJ. School environments and physical activity: The development and testing of an audit tool. Health Place. 2010;16(5):776-83.

16. Katzmarzyk PT, Barreira TV, Broyles ST, Champagne CM, Chaput JP, Fogelholm M, et al. The International Study of Childhood Obesity, Lifestyle and the Environment (ISCOLE): design and methods. BMC Public Health. 2013;13:900.

17. Prado CV. Ambiente escolar e promoção da atividade física na escola: implicações para os níveis de atividade física de adolescentes de Curitiba, PR [dissertação de Mestrado]. Curitiba: Universidade Federal do Paraná; 2014.

18. Lounsbery MA, McKenzie TL, Morrow JR, Jr, Holt KA, Budnar RG. School physical activity policy assessment. J Phys Act Health. 2013;10(4):496-503.

19. Wechsler H, Devereaux RS, Davis M, Collins J. Using the school environment to promote physical activity and healthy eating. Prev Med. 2000;31(2):121-S37.

20. Harrison F, Jones AP. A framework for understanding school based physical environmental influences on childhood obesity. Health Place. 2012;18(3):639-48.

21. Magalhães CHF, Martineli TAP. Soluções formais no enfrentamento dos problemas da prática escolar. O estranhamento dos professores de educação física escolar. Motriviv. 2011;23(36):214-35.
22. Damazio MS, Silva MFP. O ensino da educação física e o espaço físico em questão. Pensar a Prática. 2008;11(2):189-96.

23. Tenório MCM, Tassitano RM, Lima MC. Conhecendo o ambiente escolar para as aulas de educação física: existe diferença entre as escolas? Rev Bras Ativ Fis Saúde. 2012;17(4):307-13.

24. Kirby J, Levin KA, Inchley J. Associations between the school environment and adolescent girls' physical activity. Health Educ Res. 2012;27(1):101-14.

25. Haug E, Torsheim T, Sallis JF, Samdal O. The characteristics of the outdoor school environment associated with physical activity. Health Edu Res. 2010;25(2):248-56.

26. Reznik M, Wylie-Rosett J, Kim M, Ozuah PO. Physical activity during school in urban minority kindergarten and first-grade students. Pediatrics. 2013;131(1):81-7.

27. Diretrizes em educação física de qualidade (EFQ) para gestores de políticas. Brasília; UNESCO; 2015. [citado 2018 jun 02]. Disponível em: http://www.unesco.org/new/ pt/brasilia/about-this-office/single-view/news/quality_ physical_education_qpe_guidelines_for_policy_make/.

28. Ridgers ND, Stratton G, Fairclough SJ, Twisk JW. Longterm effects of a playground markings and physical structures on children's recess physical activity levels. Prev Med. 2007;44(5):393-7.

29. Brasil. Ministério da Saúde. Secretaria de Vigilância Sanitária em Saúde. Departamento de Análise de Situação em Saúde. Avaliação de efetividade de programas de educação física no Brasil Brasília; 2011:182 p.

30. Escalante Y, Garcia-Hermoso A, Backx K, Saavedra JM. Playground designs to increase physical activity levels during school recess: a systematic review. Health Educ Behav. 2014;41(2):138-44. 